\title{
Estudo comparativo da eficácia de tratamentos para a alopecia androgenética por meio da técnica intradermoterapia capilar: minoxidil e finasterida
}

\author{
Comparative study of the effectiveness of treatments for androgenetic alopecia through the \\ capillary intradermotherapy technique: minoxidil and finasteride \\ Estudio comparativo de la eficacia de tratamientos para la alopecia androgenética mediante la \\ técnica de intradermoterapia capilar: minoxidil y finasterida
}

Recebido: 28/07/2021 | Revisado: 03/08/2021 | Aceito: 04/08/2021 | Publicado: 09/08/2021

\author{
Marcus Winícius Mendes Formiga \\ ORCID: https://orcid.org/0000-0003-1723-6637 \\ Centro Universitário de Patos, Brasil \\ E-mail: marcusformiga@med.fiponline.edu.br \\ Milena Nunes Alves de Sousa \\ ORCID: https://orcid.org/0000-0001-8327-9147 \\ Centro Universitário de Patos, Brasil \\ E-mail: milenanunes@fiponline.edu.br \\ Lívio e Vasconcelos do Egypto \\ ORCID: https://orcid.org/0000-0001-7804-4116 \\ Centro Universitário de Patos, Brasil \\ E-mail: livioegypto@hotmail.com
}

\begin{abstract}
Resumo
Objetivo: comparar resultados relevantes acerca da eficácia do uso do Minoxidil, fatores de crescimento e algumas vitaminas com a associação Finasterida, fatores de crescimento e vitaminas pela técnica intradermoterapia capilar para o tratamento da Alopecia Androgenética. Métodos: A pesquisa foi realizada em uma clínica privada no município de Patos-PB. Foram avaliados dez pacientes com quadro de Alopecia Androgenética, divididos em dois grupos com cinco pessoas cada, conforme características semelhantes. O Grupo A recebeu a intradermoterapia capilar com Minoxidil, fatores de crescimento e vitaminas, enquanto o Grupo B recebeu a Finasterida, fatores de crescimento e vitaminas. Os critérios utilizados para comparação foram avaliados pelo questionário de evolução médica, levando em consideração o aumento ou a diminuição do volume capilar e a diminuição da alopecia. Após isso, os dados foram sistematizados e confrontados com a literatura atualizada sobre a temática proposta. Resultados: Foi ilustrada uma leve superioridade relacionada ao aumento do volume capilar dos pacientes do grupo A. No que tange à queda capilar, os resultados se mantiveram praticamente semelhantes entre os pacientes dos dois grupos. Conclusão: Há a necessidade de estudos com maior volume amostral, visando um resultado mais fidedigno em busca de um efeito clínico mais significativo para essa enfermidade.
\end{abstract}

Palavras-chave: Alopecia androgenética; Intradermoterapia capilar; Minoxidil; Finasterida.

\begin{abstract}
Objective: to compare relevant results regarding the effectiveness of the use of Minoxidil, growth factors and some vitamins with the association Finasteride, growth factors and vitamins by the capillary intradermotherapy technique for the treatment of Androgenetic Alopecia. Methods: The research was carried out in a private clinic in the city of Patos-PB. Ten patients with Androgenetic Alopecia were evaluated, divided into two groups of five people each, according to similar characteristics. Group A received capillary intradermotherapy with Minoxidil, growth factors and vitamins, while Group B received Finasteride, growth factors and vitamins. The criteria used for comparison were evaluated by the medical evolution questionnaire, taking into account the increase or decrease in capillary volume and the decrease in alopecia. After that, the data were systematized and compared with the updated literature on the proposed theme. Results: A slight superiority related to the increase in capillary volume of patients in group A was illustrated. With regard to capillary drop, the results remained practically similar between patients in both groups. Conclusion: There is a need for studies with a larger sample size, aiming at a more reliable result in search of a more significant clinical effect for this disease.
\end{abstract}

Keywords: Androgenetic alopecia; Capillary intradermotherapy; Minoxidil; Finasteride. 


\begin{abstract}
Resumen
Objetivo: comparar resultados relevantes sobre la efectividad del uso de Minoxidil, factores de crecimiento y algunas vitaminas con la asociación Finasteride, factores de crecimiento y vitaminas por la técnica de intradermoterapia capilar para el tratamiento de la Alopecia Androgenética. Métodos: La investigación se llevó a cabo en una clínica privada de la ciudad de Patos-PB. Se evaluaron diez pacientes con Alopecia Androgenética, divididos en dos grupos de cinco personas cada uno, según características similares. El Grupo A recibió intradermoterapia capilar con Minoxidil, factores de crecimiento y vitaminas, mientras que el Grupo B recibió Finasteride, factores de crecimiento y vitaminas. Los criterios utilizados para la comparación se evaluaron mediante el cuestionario de evolución médica, teniendo en cuenta el aumento o disminución del volumen capilar y la disminución de la alopecia. Posteriormente, los datos fueron sistematizados y comparados con la literatura actualizada sobre el tema propuesto. Resultados: Se ilustró una ligera superioridad relacionada con el aumento de volumen capilar de los pacientes del grupo A. En cuanto a la caída capilar, los resultados se mantuvieron prácticamente similares entre los pacientes de ambos grupos. Conclusión: Se necesitan estudios con mayor tamaño muestral, con el objetivo de obtener un resultado más confiable en la búsqueda de un efecto clínico más significativo para esta enfermedad.
\end{abstract}

Palabras clave: Alopecia androgenética; Intradermoterapia capilar; Minoxidil; Finasteride.

\title{
1. Introdução
}

Os cabelos desempenham uma função imprescindível na vida do ser humano. Compõe-se de um órgão envolvido na percepção sensorial, com funções diferentes, como proteção química, mecânica e orgânica (termorregulação, defesa física, renovação e regeneração tecidual), sendo muito utilizado como um meio de comunicação psicossocial (Nantes et al., 2018; Sei \& Brandão, 2020).

Anatomicamente, é definido como um pelo que está aderido a uma glândula sebácea, o folículo piloso. Este, passa pelo ciclo biológico do pelo que é dividido em três fases principais e bem distintas ao longo do seu desenvolvimento: a anágena ou de crescimento, a catágena ou de involução e a telógena ou de repouso, culminando com a sua regeneração em sucessivos ciclos, que ocorrem após a queda e substituição por um novo fio anágeno. Tais alterações culminam em distúrbios de crescimento, a exemplo da Alopecia (Pereira et al., 2016; Louzada, Mendes \& Leite, 2019).

Segundo a Sociedade Brasileira de Dermatologia dentre a procura nos consultórios dermatológicos, a queixa de alopecia está entre as dez patologias mais frequentes. A alopecia é uma doença dermatológica que acomete os folículos pilosos, ocorrendo diminuição gradativa dos fios no couro cabeludo (Pereira, 2018; Louzada, Mendes \& Leite, 2019).

Em pacientes com diagnóstico de alopecia androgenética, ocorre uma alteração no ciclo biológico do pelo, responsável pelo processo de miniaturização de todo o aparelho folicular. A fase anágena ou de crescimento tem sua duração reduzida, enquanto a persistência na fase telógena permanece constante ou prolongada, levando a transformação de cabelos terminais em velo (pelo pequeno) com consequente perda da densidade do cabelo (Keaney, 2015; Caserini et al., 2016; Rossi et al., 2016).

A alopecia androgenética (AAG) é a causa mais frequente de alopecia em ambos os sexos. Acredita-se que entre os homens acima de 50 anos, 50\% manifestam algum grau de calvície. Entretanto, entre as mulheres, os números podem variar, sendo o acometimento aproximado de 30\%, intensificando-se em torno dos 70 anos. Não está correlacionada diretamente com nenhuma etnia específica, todavia, existe uma predisposição menor em asiáticos e afrodescendentes em relação aos caucasianos (Mulinari-Brenner, Seidel \& Hepp 2011; Adil \& Godwin, 2017; Lee, Juhasz, Mobasher, Ekelem \& Mesinkovska, 2018).

A queda de cabelo pode afetar a qualidade de vida do paciente, pois gera prejuízos estéticos e, consequentemente, acarreta um desconforto emocional direcionando estes pacientes a buscarem o tratamento (Machado \& Marques, 2017; Louzada, Mendes \& Leite, 2019). 
Atualmente existem diversos tratamentos disponíveis para alopecia androgenética no mercado, destacando-se a intradermoterapia capilar ou mesoterapia. Está é considerada uma terapia alternativa não cirúrgica, menos invasiva e que melhora a microcirculação local, desacelera a involução dos folículos pilosos e impulsiona o seu desenvolvimento através da introdução de substâncias ativas por via intradérmica em uma profundidade de cerca de $4 \mathrm{~mm}$ diretamente no couro cabeludo, proporcionando assim, uma alta concentração e absorção da medicação no bulbo folicular que é a região mais profunda do folículo (Hillmann et al., 2015; Frano \& Tassinary, 2018).

Tendo em vista o papel fundamental e a relevância do cabelo na sociedade, o objetivo desse estudo é comparar resultados relevantes acerca da eficácia do uso do Minoxidil, fatores de crescimento e algumas vitaminas com a associação Finasterida, fatores de crescimento e vitaminas pela técnica intradermoterapia capilar para o tratamento da Alopecia Androgenética em pacientes de ambos os sexos.

\section{Metodologia}

Ensaio clínico randomizado duplo-cego e prospectivo com abordagem quantitativa. A pesquisa foi realizada em uma clínica privada, localizada no município de Patos-PB. Foram avaliados dez pacientes, com história familiar ou não de alopecia, de ambos os sexos, sem limitação de faixa etária e com quadro de Alopecia Androgenética, sendo divididos em dois grupos com cinco pessoas cada de modo confidencial por meio de sorteio, conforme características semelhantes (Estrela, 2018).

O grupo A recebeu a intradermoterapia com Minoxidil, Fatores de Crescimento e algumas vitaminas; o grupo B a intervenção foi a associação entre a Finasterida, Fatores de Crescimento e vitaminas. Foram excluídos pacientes que não conseguiram seguir estritamente o protocolo ou que apresentaram efeitos adversos à medicação.

O protocolo clínico foi aprovado pelo Comitê de Ética do Centro Universitário de Patos (UNIFIP), CAAE 39877020.4.0000.5181/4.490.961 e seguiu a Resolução nº 466/2012 do Ministério da Saúde, respeitando todos os aspectos éticos de pesquisas com seres humanos. Todos os pacientes incluídos assinaram o Termo de Consentimento Livre e Esclarecido (TCLE) antes de serem triados para o estudo. A terapia foi realizada no período de Março à Junho de 2021.

Os critérios utilizados para comparação foram avaliados pelo questionário de evolução médica, levando em consideração o aumento ou a diminuição do volume de cabelo, bem como, a diminuição da alopecia. Após isso, os dados foram sistematizados, analisados e confrontados com a literatura atualizada sobre a temática em questão.

\section{Resultados}

Entre os pacientes selecionados para a pesquisa, segundo os dados elucidados na Tabela 1, 40\% referiu tempo de doença de 1 a 5 anos, enquanto isso, 60\% acusaram que possuem a doença há mais de 5 anos. Já com relação à história familiar, $80 \%$ dos pacientes relataram história familiar de primeiro grau de calvície, fora estes, $10 \%$ relataram história em parentes de segundo grau e os outros $10 \%$ não confirmaram histórico familiar. 
Tabela 1: Tempo de doença e história familiar dos pacientes por grupo.

\begin{tabular}{lll}
\hline Pacientes & Tempo de doença & História familiar \\
\hline GRUPO A & 1 a 5 anos & Segundo grau \\
Paciente 1 & Mais de 5 anos & Primeiro grau \\
\hline Paciente 2 & 1 a 5 anos & Ausente \\
Paciente 3 & 1 a 5 anos & Primeiro grau \\
\hline Paciente 4 & Mais de 5 anos & Primeiro grau \\
\hline Paciente 5 & & \\
GRUPO B & Mais de 5 anos & Primeiro grau \\
\hline Paciente 6 & 1 a 5 anos & Primeiro grau \\
Paciente 7 & Mais de 5 anos & Primeiro grau \\
\hline Paciente 8 & Mais de 5 anos & Primeiro grau \\
\hline Paciente 9 & Mais de 5 anos & Primeiro grau \\
\hline Paciente 10 & & \\
\hline
\end{tabular}

Fonte: Autores (2021).

De acordo com a resposta acerca do volume dos cabelos, os pacientes do Grupo A (Gráfico 1), que realizaram a intradermoterapia com Minoxidil, fatores de crescimento e vitaminas, em $60 \%$ dos pacientes que houve um aumento leve do volume do cabelo, em $20 \%$ desses ocorreu um aumento moderado do volume capilar e outros $20 \%$ um aumento acentuado do volume do cabelo. Já entre os pacientes do Grupo B (Gráfico 2), os quais realizaram a intradermoterapia com Finasterida, fatores de crescimento e vitaminas, em $60 \%$ desse ocorreu um aumento leve do volume capilar, em $20 \%$ o aumento do volume foi moderado e outros $20 \%$ não houve modificação e o volume capilar se manteve inalterado.

Gráfico 1: Escala de volume capilar Grupo A.

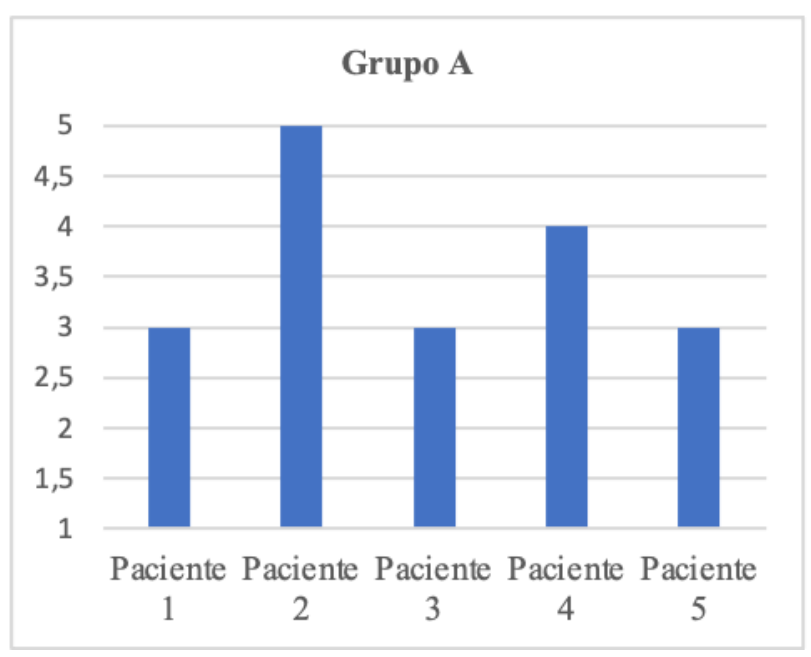

Fonte: Autores (2021).
Gráfico 2: Escala de volume capilar Grupo B.

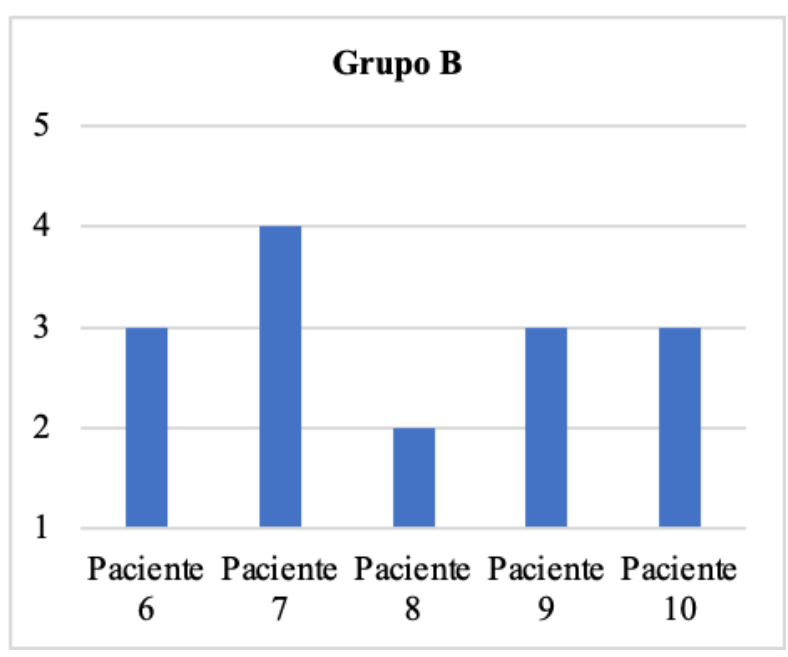

Fonte: Autores (2021).

Enquanto que, com relação à diminuição da queda de cabelos, no grupo A (Gráfico 3), foi percebido que em $100 \%$ dos pacientes da referida amostra houve diminuição da queda dos cabelos. Já no grupo B, os resultados acerca da queda capilar (Gráfico 4) mostraram que em $80 \%$ dos pacientes houve diminuição da queda de cabelos e nos $20 \%$ restantes a queda capilar se manteve inalterada. 
Gráfico 3: Escala da diminuição da queda capilar.

Gráfico 4: Escala da diminuição da queda capilar Grupo

B.

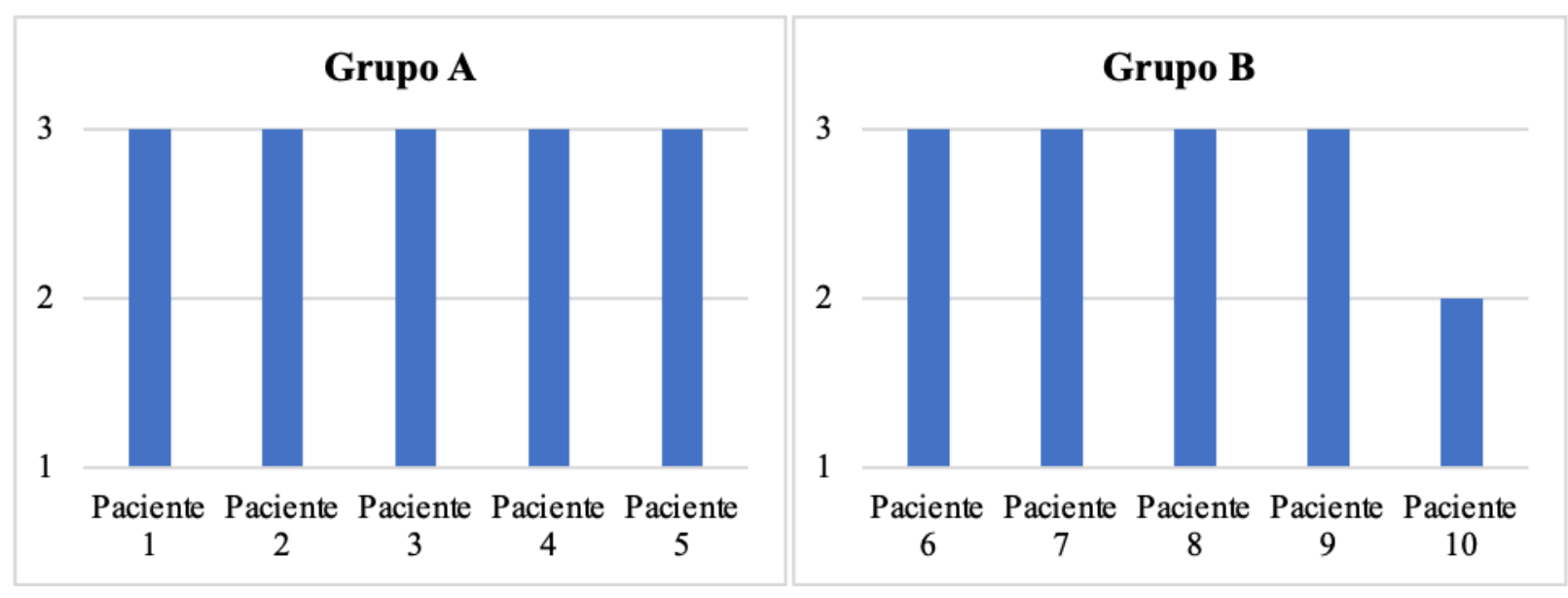

Fonte: Autores (2021).

Fonte: Autores (2021).

\section{Discussão}

Tendo em vista que a alopecia androgenética é uma condição progressiva e necessita de uma abordagem terapêutica adequada e precoce. Seu tratamento é baseado na estabilização do processo de miniaturização, buscando impedir que os cabelos já miniaturizados entrem em processo de queda irreversível, bem como diminuir a queda diária de cabelos e aumentar a densidade capilar. Esses objetivos podem ser alcançados por meio de uma dieta balanceada com ingestão adequada de proteínas e ferro, controle de peso em pacientes obesos, o que reduz a transformação periférica dos androgênios e, com isso, melhorando o perfil hormonal. Além disso, ressalta-se que a importância do tratamento de outras possíveis desordens capilares presentes, como a dermatite seborreica para o sucesso terapêutico (Dhurat et al., 2016; Pereira et al., 2016).

Com relação aos resultados atingidos com o presente estudo, foi ilustrada uma leve superioridade quando analisado o aumento do volume capilar dos pacientes do grupo A, que foram infundidos com a intradermoterapia capilar com Minoxidil, fatores de crescimento e vitaminas. Em um estudo recente realizado por Cavalcanti \& Arruda (2015), observou-se que o Minoxidil possui uma ação ainda pouco elucidada no tratamento da alopecia androgenética. Porém, sabe-se que ele atua na fase anágena com o intuito de aumentar a sua duração e, consequentemente, a densidade capilar por conta do seu mecanismo que causa um maior fluxo vascular cutâneo. Entretanto, foram notados alguns efeitos adversos como eritema local, descamação, prurido, hipotensão arterial, náuseas, fadiga, erupção cutânea, cefaleia, retenção hidrossalina com edemas e hipertricose.

Outro medicamento comumente utilizado nessa enfermidade é a Finasterida, o qual tem maior comprovação nos casos em que a alopecia se dá por uma combinação de fatores hereditários e do hormônio Di-hidrotestosterona (DHT). Esta, que age no encurtamento da fase do crescimento do cabelo e consequente diminuição do volume do cabelo. A medicação atua inibindo a enzima $5 \alpha$-redutase tipo 2 que é responsável pela transformação de testosterona em DHT, ligando-se irreversivelmente à enzima e, com isso, bloqueando a conversão da testosterona, evitando assim a miniaturização dos folículos. Entretanto, no presente estudo, a utilização dessa medicação associada a fatores de crescimento e vitaminas foi inferior quando comparada à utilização de Minoxidil, fatores de crescimento e vitaminas (Gupta, Carviel, Macleod \& Shear, 2017; Kiguradze et al., 2017; Machado \& Marques, 2017). 
Em um estudo realizado por Gupta et al. (2018) em que foi comparada a eficácia de diversos tratamentos não cirúrgicos para a alopecia androgenética, percebeu-se que a Finasterida de uso oral teve resultados superiores ao Minoxidil 5\% quanto à alopecia. No entanto, foi estimado que o Minoxidil 5\% era mais favorável ao uso, visto que os efeitos colaterais da Finasterida eram mais incômodos para a população que utilizava esse medicamento. Dentre os principais efeitos adversos, a disfunção sexual configurou-se como o de maior apreensão, havendo relatos de pacientes com esta deficiência mesmo um ano após o fim da terapêutica.

Entretanto, no presente ensaio clínico, houve comprovação de que os pacientes do Grupo B obtiveram resultados levemente inferiores aos do Grupo A, com relação ao aumento do volume capilar. Associado a isso, outra pesquisa realizada por Suchonwanit, Srisuwanwattana, Chalermroj \& Khunkhet (2018), comprovou que os inibidores da $5 \alpha$-redutase, quando utilizados via tópica, reduzia esses efeitos adversos, gerando expectativa por atuarem diretamente nos folículos pilosos alvo. Nesse estudo em questão, foi utilizado uma solução de 0,25\% de Finasterida misturada com 3\% de Minoxidil, comparada com outra solução de Minoxidil a 3\%. Notou-se que 60\% dos pacientes tratados com a mistura, apresentaram melhoria acentuada e efeitos adversos sistêmicos ou qualquer queixa de disfunção sexual não foi relatada, apenas reações locais leves no local da aplicação.

No que diz respeito à queda capilar, os resultados se mantiveram praticamente semelhantes entre os pacientes dos dois grupos. Apresentando apenas, uma queda capilar menor acentuada em um dos pacientes do Grupo B, que fez uso de Finasterida, fatores de crescimento e vitaminas.

Apesar dos achados importantes, como limitações do estudo apresentam-se a pequena amostra utilizada devido ao custo do procedimento, já que a pesquisa foi financiada pelos próprios pesquisadores, bem como pelo período de pandemia devido ao novo coravírus (COVID-19), fator também complicador ao alcance de amostra de pesquisa mais representativa.

\section{Conclusão}

De acordo com os resultados da pesquisa, é de suma importância atentar para as novas terapias que podem ser utilizadas na alopecia androgenética. Percebe-se que existem novas formas de tratamento que auxiliam tanto no aumento de volume capilar, quanto na diminuição da queda de cabelos. Em suma, de acordo com os dados colhidos, percebeu-se sutil vantagem da intradermoterapia capilar com Minoxidil, fatores de crescimento e vitaminas em detrimento à utilização de Finasterida, fatores de crescimento e vitaminas, quando comparados o aumento do volume capilar e diminuição da queda de cabelos. Entretanto, há a necessidade de estudos com maior volume amostral, visando um resultado mais fidedigno acerca de qual terapia seria a mais impactante em busca de um efeito clínico mais significativo para essa enfermidade.

Ademais, fazem-se necessários novos estudos comparativos utilizando outras drogas relacionadas à alopecia androgenética, bem como, a abordagem terapêutica aos outros tipos de alopecia.

\section{Referências}

Adil A., \& Godwin M. (2017). The effectiveness of treatments for androgenetic alopecia: a systematic review and meta-analysis. Journal Of The American Academy Of Dermatology, 77(1), 136-141.

Caserini, M., Radicioni, M., Leuratti, C., Terragni, E., Iorizzo, M., \& Palmieri, R. (2016). Effects of a novel finasteride $0.25 \%$ topical solution on scalp and serum dihydrotestosterone in healthy men with androgenetic alopecia. International journal of clinical pharmacology and therapeutics, 54(1), 19-27. https://doi.org/10.5414/CP202467

Cavalcanti, C. P., \& Arruda T. A. (2015). Protocolos de tratamento da alopecia: uma revisão. Trabalho de Conclusão de Curso (Graduação em Farmácia) Universidade Estadual da Paraíba, Campina Grande, 30 p.

Dhurat, R., Chitallia, J., May, T. W., Jayaraaman, A. M., Madhukara, J., Anandan, S., Vaidya, P., \& Klenk, A. (2017). An Open-Label Randomized Multicenter Study Assessing the Noninferiority of a Caffeine-Based Topical Liquid 0.2\% versus Minoxidil 5\% Solution in Male Androgenetic Alopecia. Skin pharmacology and physiology, 30(6), 298-305. https://doi.org/10.1159/000481141 
Estrela, C. (2018). Metodologia Científica: Ciência, Ensino, Pesquisa. Editora Artes Médicas.

Frano, J., \& Tassinary J. (2018). Revisão bibliográfica dos principais recursos terapêuticos utilizados no tratamento da alopécia androgenética. Revista Destaques Acadêmicos, 10(3): 8-14.

Hillmann, K., Garcia Bartels, N., Kottner, J., Stroux, A., Canfield, D., \& Blume-Peytavi, U. (2015). A Single-Centre, Randomized, Double-Blind, PlaceboControlled Clinical Trial to Investigate the Efficacy and Safety of Minoxidil Topical Foam in Frontotemporal and Vertex Androgenetic Alopecia in Men. Skin pharmacology and physiology, 28(5), 236-244. https://doi.org/10.1159/000375320

Gupta, A. K., Carviel, J., Macleod, M. A., \& Shear, N. (2017). Assessing finasteride-associated sexual dysfunction using the FAERS database. Journal Of The European Academy Of Dermatology And Venereology, 31(6), 1069-1075. http://dx.doi.org/10.1111/jdv.14223.

Gupta, A. K., Mays, R. R., Dotzert, M. S., Versteeg, S. G., Shear, N. H., \& Piguet, V. (2018). Efficacy of non-surgical treatments for androgenetic alopecia: a systematic review and network meta-analysis. Journal of the European Academy of Dermatology and Venereology: JEADV, 32(12), 2112-2125. https://doi.org/10.1111/jdv.15081

Keaney T. (2015). Emerging therapies for androgenetic alopecia. Journal of Drugs in Dermatology, 14(9): 1036-1040.

Kiguradze, T., Temps, W. H., Yarnold, P. R., Cashy, J., Brannigan, R. E., Nardone, B., Micali, G., West, D. P., \& Belknap, S. M. (2017). Persistent erectile dysfunction in men exposed to the 5 $\alpha$-reductase inhibitors, finasteride, or dutasteride. PeerJ, 5, e3020. https://doi.org/10.7717/peerj.3020

Lee, S. W., Juhasz, M., Mobasher, P., Ekelem, C., \& Mesinkovska, N. A. (2018). A Systematic Review of Topical Finasteride in the Treatment of Androgenetic Alopecia in Men and Women. Journal of drugs in dermatology: JDD, 17(4), 457-463.

Louzada, L. M., Mendes, R. de F., \& Leite, S. A. (2019). Alopécia Androgenética: principais abordagens terapêuticas. Trabalho de Conclusão de Curso (Graduação em Medicina) - Centro Universitário UNIFACIG, 2019, 22 p.

Machado I. O. C. C., \& Marques L. T. (2017). Calvície e alopecia: revisão bibliográfica. Dissertação (Mestrado em Ciências Farmacêuticas) - Universidade Lusófona de Humanidade e Tecnologias, 65 p.

Mulinari-Brenner F., Seidel, G., \& Hepp, T. (2011). Understanding androgenetic alopecia. Surgical \& Cosmetic Dermatology, 3(4): 329-37.

Nantes, M. C., De Paiva, N. S., Soares, A. L. F., Santos, J. L., Eler, J. F. de C., \& Lopes, L. de A. (2018). Action of minoxidil and finasteride through intradermotherapy in the treatment of androgenetic alopecia. Brazilian Journal of Surgery and Clinical Research, 24(2): 166-175.

Pereira, J. M. et al. (2016). Tratado das doenças dos cabelos e do couro cabeludo: Tricologia. Di Livros Editora, 503 p.

Pereira, L. A. (2018). Principais tipos de alopecias não cicatriciais e suas Fisiopatogenias. Revista Estética em Movimento, 1:1-18

Rossi, A., Anzalone, A., Fortuna, M. C., Caro, G., Garelli, V., Pranteda, G., \& Carlesimo, M. (2016). Multi-therapies in androgenetic alopecia: review and clinical experiences. Dermatologic therapy, 29(6), 424-432. https://doi.org/10.1111/dth.12390

Sei, M. C. K., \& Brandão B. J. F. (2020). Use of Mesotherapy for Androgenetic Alopecia: a literature review. BWS Journal, 3 , e20060080: 1-7.

Suchonwanit, P., Srisuwanwattana, P., Chalermroj, N., \& Khunkhet, S. (2018). A randomized, double-blind controlled study of the efficacy and safety of topical solution of $0.25 \%$ finasteride admixed with $3 \%$ minoxidil vs. $3 \%$ minoxidil solution in the treatment of male androgenetic alopecia. Journal of the European Academy of Dermatology and Venereology: JEADV, 32(12), 2257-2263. https://doi.org/10.1111/jdv.15171 